\title{
Covariant Analysis of Gravitational Waves in a Cosmological Context
}

\author{
Peter K. S. Dunsby ${ }^{1,2}$, Bruce A. C. C. Bassett ${ }^{3}$ and George F. R. Ellis ${ }^{1}$ \\ 1. Department of Mathematics and Applied Mathematics, \\ University of Cape Town, South Africa. \\ 2. Department of Mathematics, Statistics and Computing Science, \\ Dalhousie University, Halifax, Nova Scotia, Canada. \\ 3. Scuola Internazionale Superiore de Studi Avanzati, \\ Strada Costiera 11, Miramare, 34014, Italy.
}

June 13, 2018

\begin{abstract}
The propagation of gravitational waves or tensor perturbations in a perturbed Friedmann-Robertson-Walker universe filled with a perfect fluid is re-examined. It is shown that while the shear and magnetic part of the Weyl tensor satisfy linear, homogeneous second order wave equations, for perfect fluids with a $\gamma$ - law equation of state satisfying $\frac{2}{3}<\gamma<2$, the electric part of the Weyl tensor satisfies a linear homogeneous third order equation. Solutions to these equations are obtained for a flat Friedmann Robertson - Walker background and we discuss implications of this result.
\end{abstract}

\section{Introduction}

Both the covariant definition and behaviour of gravitational waves and the dual question of the physical interpretation of the electric $\left(E_{a b}\right)$ and magnetic $\left(H_{a b}\right)$ parts of the Weyl tensor are subjects of debate at present. In particular the question arises whether it is possible to neglect the magnetic part of the Weyl tensor in the Newtonian limit, and whether this is equivalent to neglecting the gravitational wave contribution to cosmological perturbations. It is the aim in this paper to extend this debate by comparing the analysis of gravitational waves, set in a cosmological context, using the covariant approach on the one hand and the more standard methods based on metric perturbations on the other.

In the standard approach a second order propagation equation for the amplitude of the tensor perturbations is derived either via the variation of the 
Einstein - Hilbert action after expansion to second order, or by directly linearizing the Einstein field equations. For example in the Bardeen formalism [1] the following equation of motion is obtained for the first order gauge-invariant amplitude of the tensor perturbation $H_{T}^{(2)}$, in the absence of anisotropic stress perturbations: 1

$$
\ddot{H}_{T}^{(2)}+\frac{2 \dot{\ell}}{\ell} \dot{H}_{T}^{(2)}+\left(k^{2}+2 K\right) H_{T}^{(2)}=0,
$$

where $\ell$ is the cosmological scale factor, $K$ is the spatial curvature of the background Friedmann-Robertson-Walker (FRW) model, and $k$ is the physical wavenumber when $K=0$ 12]. The full metric tensor perturbation is $H_{T}^{(2)} Q_{\mu \nu}^{(2)}$, where $Q^{(2)}{ }_{\mu \nu}$ are eigenfunctions (polarization tensors) of the tensor Helmholtz equation on the background spatial sections. These eigenfunctions have only two degrees of freedom after imposing the transverse $\left(Q_{; \nu}^{(2) \mu \nu}=0\right)$ and traceless $\left(Q^{(2) \mu}{ }_{\mu}=0\right)$ conditions.

By contrast, the covariant approach focuses on either the electric or magnetic parts of the Weyl tensor [11, 5], and their propagation equations when linearized about a FRW model (which include waves propagating in a flat-space background as a limiting case). In this paper we will present a rather unusual result: while in a vacuum both $E_{a b}$ and $H_{a b}$ satisfy second order wave equations, when the spacetime is non-empty, and the matter is described by a barotropic perfect fluid with the equation of state $p=(\gamma-1) \mu$ satisfying $\frac{2}{3}<\gamma<2$, we obtain a third order equation for $E_{a b}$ but a second order equations for both $H_{a b}$ and the shear tensor $\sigma_{a b}$. This is somewhat like considering Maxwell's equations with sources [8], except in the gravitational case, the fundamental difference is that the source can never be turned off if there is matter present.

This paper is organized as follows: in section 2 we outline the covariant characterization of gravitational waves. In section 3 we present differential equations in closed form which describe the propagation of gravitational waves on a FRW background with a perfect fluid source. Solutions to these equations for a flat background are given in section 4 and the paper ends with a discussion of the cosmological context of our results.

\subsection{Notation and Conventions}

Conventions are taken to be the same as in [5] and we take $c=1$. It is assumed that Einstein's equations take the form $G_{a b}=\kappa T_{a b}$ where $G_{a b}$ is the Einstein tensor, $\kappa=8 \pi G$ is the gravitational constant and $T_{a b}$ is the energy - momentum tensor of the matter, which is taken to be a perfect fluid. We use the standard kinematical decomposition of the first covariant derivative of $u^{a}$, 年, a "dot" denotes the covariant derivative along the fluid flow lines and ${ }^{(3)} \nabla_{a}$ corresponds to the 3 -dimensional covariant derivative defined by totally projecting

\footnotetext{
${ }^{1}$ The equation for tensor metric perturbations was first derived by Lifshitz in 1946 [14.
} 
the covariant derivative orthogonal to $u^{a}$, so for example

$$
\dot{T}_{a b}=T_{a b ; c} u^{c}, \quad{ }^{(3)} \nabla_{a} T_{b c}=h^{s}{ }_{a} h^{t}{ }_{b} h^{u}{ }_{c} T_{t u ; s},
$$

where

$$
h_{a b}=g_{a b}+u_{a} u_{b}
$$

is the usual 3 - dimensional projection tensor orthogonal to $u^{a}$.

A Robertson-Walker background geometry is characterized by the shear, vorticity, and acceleration, together with their covariant derivatives, being at most first order, while the energy density and expansion are zero order, and the pressure can be so also [6]. It follows that the electric and magnetic parts of the Weyl tensor:

$$
E_{a b}=C_{a c b d} u^{c} u^{d} \quad H_{a b}=\frac{1}{2} C_{a c s t} \eta^{s t}{ }_{b d} u^{c} u^{d}
$$

are also at most first order. We will assume that in a cosmological context, $(\mu+p) \Theta>0$.

\section{The covariant approach to gravitational waves}

In the covariant approach, the study of tensor perturbations was first considered by Hawking [11. In his paper, the electric part of the Weyl tensor was used as the variable to characterize them. Later it was suggested that the Magnetic part of the Weyl tensor is a better choice because it has no analogue in Newtonian theory where gravity is propagated instantaneously. Hence the Magnetic part obviously plays an important role in describing gravitational waves, but given the correspondence with electromagnetism [8], where neither the electric nor magnetic fields provide a complete description of EM waves, we suggest that both electric and magnetic parts of the Weyl tensor are required for a full understanding of tensor perturbations. Indeed it is their curls that characterize gravitational waves, as we will see below.

The fully nonlinear evolution equations for $E_{a b}$ and $H_{a b}$, with the matter source described by a perfect fluid, are given by [5]:

$$
\begin{gathered}
h_{a}^{m}{ }_{a} h_{c}^{t} \dot{E}^{a c}+h^{(m}{ }_{a} \eta^{r) t s d} u_{r} H_{s ; d}^{a}-2 H^{(t}{ }_{q} \eta^{m) b p q} u_{b} \dot{u}_{p}+\Theta E^{m t} \\
+h^{m t}\left(\sigma^{a b} E_{a b}\right)-3 E^{(m}{ }_{s} \sigma^{t) s}-E^{(m}{ }_{s} \omega^{t) s}=-\frac{1}{2}(\mu+p) \sigma^{t m}
\end{gathered}
$$

and

$$
\begin{aligned}
& h^{m a} h^{t c} \dot{H}_{a c}-h_{a}{ }^{(m} \eta^{r) t s d} u_{r} E^{a}{ }_{s ; d}+2 E_{q}^{(t} \eta^{m) b p q} u_{b} \dot{u}_{p} \\
& \quad+h^{m t}\left(\sigma^{a b} H_{a b}\right)+\Theta H^{m t}-3 H^{(m}{ }_{s} \sigma^{t) s}-H^{(m}{ }_{s} \omega^{t) s}=0 .
\end{aligned}
$$

Notice that just as in the electromagnetic case, the only difference between these equations is in the sign of the second and third terms, and the source term (here, 
the shear) coupled to the energy density and pressure in the $\dot{E}_{a b}$ equation. Once linearized about a FRW background, these equations become:

$$
\dot{E}_{a b}+\Theta E_{a b}+{ }^{(3)} \nabla^{e} H_{(a}^{d} \eta_{b) c d e} u^{c}+\frac{1}{2} \kappa(\mu+p) \sigma_{a b}=0
$$

and

$$
\dot{H}_{a b}+\Theta H_{a b}-{ }^{(3)} \nabla^{e} E^{d}{ }_{(a} \eta_{b) c d e} u^{c}=0 .
$$

On taking the time-derivative of the first and substituting from the second, it is the curl $E$ and curl $H$ terms that give rise to traveling gravitational waves, in analogy with the propagation of electromagnetic waves.

It is immediately clear from the above that (given the FRW background evolution, which determines the zero-order coefficients in the equations), these equations by themselves do not close up in general, because of the last term in (87). One has to add to them the shear evolution equation, which in linearized form is

$$
\dot{\sigma}_{a b}={ }^{(3)} \nabla_{(a} \dot{u}_{b)}-\frac{1}{3} h_{a b} \dot{u}_{; c}^{c}-\frac{2}{3} \Theta \sigma_{a b}-E_{a b} .
$$

This is crucial in leading to the third-order equation for $E_{a b}$, on taking the time derivative of (7).

One must also be aware of the constraint equations that have to be satisfied. In particular, after linearization we find the 'div E' and 'div H' equations

$$
\begin{aligned}
E_{; b}^{a b} & =\frac{1}{3} \kappa X^{a} \\
H_{; b}^{a b} & =(\mu+p) \omega^{a},
\end{aligned}
$$

where $X^{a} \equiv h^{a b} \mu_{, b}$ is the spatial gradient of the energy density [6] and $\omega^{a}$ is the vorticity vector, which are the remaining 'Maxwell-like' equations for $E_{a b}$ and $H_{a b}$.

\section{Closed evolution equations for linear gravita- tional waves}

In linear perturbations of FRW models, pure tensor perturbations are characterized by requiring that the following two quantities vanish to first order 111, 3:

$$
\begin{aligned}
& X^{a}=0 \quad \Rightarrow \quad E_{; b}^{a b}=0, \\
& \omega^{a}=0 \quad \Rightarrow \quad H_{; b}^{a b}=0,
\end{aligned}
$$

the first one excluding scalar (density) perturbations and the second one, vector (rotational) perturbations. The conditions that the terms on the right hand side vanish, following from (10,11), are analogous to the transverse condition on 
tensor perturbations in the metric approach, In addition, we notice that since the Weyl tensor is the trace-free part of the Riemann tensor, both $E_{a b}$ and $H_{a b}$ are trace-free, again like the tensor perturbations of the Bardeen approach.

Given the assumed equation of state, these conditions also imply that the spatial gradients of the pressure and expansion and the acceleration vanish:

$$
Y_{a} \equiv h^{b}{ }_{a} p_{, b}=0, \quad Z_{a} \equiv h^{b}{ }_{a} \Theta_{, b}=0, \quad \dot{u}_{a}=0,
$$

the first following from the equation of state, the second from the evolution equations for $X^{a}$ (see [6]), and the third from the momentum conservation equations. Together with (10,11) these characterize tensor perturbations.

Once conditions (12) and (13) have been imposed, one might suspect that both $E_{a b}$ and $H_{a b}$ would show the usual symmetry, i.e. that their propagation equations are the same under the substitution $E_{a b} \Leftrightarrow H_{a b}$. However as we will see below, this is not the case in general. In fact, we will demonstrate that it is only true when the equation of state of the background spacetime satisfies very special conditions. In general, as mentioned in the introduction, the equations for $E_{a b}$ and $H_{a b}$ are not even of the same order, the former satisfies a third order equation while the latter satisfies a second order one. In order to see this, let us begin by considering the linearized second-order equation for tensor perturbations, obtained by taking the time derivative of (7) and substituting from (8):

$$
\Delta E_{a b}+\frac{7}{3} \Theta \dot{E}_{a b}+\left(\frac{2}{3} \Theta^{2}-2 p\right) E_{a b}-\frac{1}{6} \mu \gamma\left[(3 \gamma-2) \Theta-\frac{3 \dot{\gamma}}{\gamma}\right] \sigma_{a b}=0,
$$

where $\Delta E_{a b}=\ddot{E}_{a b}-{ }^{(3)} \nabla^{2} E_{a b}$. By differentiating this equation, using the linearised shear evolution equation (9) specialized to tensor perturbations:

$$
\dot{\sigma}_{a b}=-\frac{2}{3} \Theta \sigma_{a b}-E_{a b},
$$

to eliminate $\dot{\sigma}_{a b}$, and using (15) again to eliminate the term in $\sigma_{a b}$, we can eliminate the remaining shear dependence, however this still doesn't give an equation that is closed. This situation can be rectified if we perform a harmonic decomposition, expanding $E_{a b}$ in terms of eigenfunctions of the Laplace-Beltrami operator of the background FRW model: $E_{a b}=\sum E_{(k)} Q^{(k)}{ }_{a b}$. In this way we can replace the Laplacian term ${ }^{(3)} \nabla^{2}$ in (15) by $k^{2} / \ell^{2}$ where $k$ is the physical wave number if the background is flat [12]. This gives us the closed, third order evolution equation for the harmonic components of $E_{a b}$ :

$$
\begin{aligned}
\dddot{E}_{(k)} & +\left[3 \Theta-\frac{\dot{B}}{B}\right] \ddot{E}_{(k)}+\left[\frac{7}{9} \Theta^{2}-\frac{7}{6}(\mu+3 p)+A-\frac{7}{3} \frac{\Theta \dot{B}}{B}\right] \dot{E}_{(k)} \\
& +\left[\dot{A}-\frac{A \dot{B}}{B}+\frac{2}{3} \Theta A-B\right] E_{(k)}=0
\end{aligned}
$$

where

$$
A=\frac{2}{3} \Theta^{2}-2 p+\frac{k^{2}}{\ell^{2}}
$$


and

$$
B=-\frac{1}{6} \mu \gamma\left[(3 \gamma-2) \Theta-\frac{3 \dot{\gamma}}{\gamma}\right] .
$$

In contrast, the propagation equation for $H_{a b}$ is relatively simple:

$$
\Delta H_{a b}+\frac{7}{3} \Theta \dot{H}_{a b}+\left(\frac{2}{3} \Theta^{2}-2 p\right) H_{a b}=0 .
$$

The reason why the equation for $H_{a b}$ is second order is due to the constraint equation [5] (with vanishing vorticity):

$$
H_{a b}=-{ }^{(3)} \nabla^{e} \sigma^{d}{ }_{(a} \eta_{b) c d e} u^{c} .
$$

This allows the shear term - arising on taking the time derivative of (8) and substituting from (77) - to be replaced in terms of $H_{a b}$. In the case of the equation for $E_{a b}(15)$, the shear term cannot be removed without differentiating again, leading to the higher order equation.

The shear also satisfies a second order equation. This follows by differentiating equation (16) and using (7) and (21) to substitute for $E_{a b}$ and $H_{a b}$ :

$$
\Delta \sigma_{a b}+\frac{5}{3} \Theta \dot{\sigma}_{a b}+\left[\frac{1}{9} \Theta^{2}+\frac{1}{6} \mu(9 \gamma-1)\right] \sigma_{a b}=0 .
$$

By comparing the equations (15) for $E_{a b}$ and (20) for $H_{a b}$, it is clear that they will be identical in form iff the equation of state (assumed to satisfy $\gamma \mu \neq 0$ ) satisfies

$$
\dot{\gamma}-\frac{1}{3} \gamma(3 \gamma-2)=0,
$$

for then the last term in (15) will vanish. In the case of a barotropic perfect fluid where $\gamma$ is a constant, two possibilities exist: either $\gamma=0 \Leftrightarrow \mu+p=0$ corresponding to a false vacuum, already rejected, or $\gamma=\frac{2}{3}$ which represents the coasting solution and is also non-physical. If $\gamma$ is allowed to vary, then (23) will also be satisfied provided

$$
\gamma=\frac{2}{\beta-\exp \frac{2}{3} t},
$$

where $\beta$ is an arbitrary constant. However this will usually have to be rejected because it will then, for early or late times, exceed the standard bounds $0 \leq$ $d p / d \mu \leq 1$ demanded for physical reasons [5] (note that when $\gamma$ varies, it is no longer true that $d p / d \mu=\gamma$ ). For normal matter satisfying $\frac{2}{3}<\gamma<2$, the equations for $E_{a b}$ and $H_{a b}$ will be very different.

The appearance of a third order equation for the electric part of the Weyl tensor $E_{a b}$ is very surprising. First of all the standard theory discussed in section 11 gives a second order evolution equation (11), and secondly, force laws are expected to be formulated as second order evolution equations. We consider this again in section 6. Hawking's covariant analysis [11] gave a second order equation, because he assumed a vacuum condition, instead of consistently sticking to a cosmological context (as assumed here). We consider this again in section 5.1 . 


\section{Solutions for a flat FRW background}

At first glance there seems to be an inconsistency with waves mediated by $E_{a b}$ and $H_{a b}$, where one is governed by a second order equation and the other by a third order equation. However consistency of the equations has been carefully checked [3, 13. We need then to explore the nature of the solutions, and also check whether there is some hidden symmetry in the equations that makes them compatible.

For simplicity we will solve the above equations only in the case of a flat FRW background $(K=0)$. Using the conformal time variable $\eta: \frac{d \eta}{d t}=\frac{1}{\ell}$, the equations for the harmonic components of the shear and magnetic part of the Weyl tensor become

$$
\sigma_{(k)}^{\prime \prime}+\frac{4 \ell^{\prime}}{3 \ell} \sigma_{(k)}^{\prime}-\left[\frac{1}{2} \mu \ell^{2}(3 \mu-4)-k^{2}\right] \sigma_{(k)}=0,
$$

and

$$
H_{(k)}^{\prime \prime}+\frac{6 \ell^{\prime}}{\ell} H_{(k)}^{\prime}-\left[2 \mu \ell^{2}(\gamma-2)-k^{2}\right] H_{(k)}=0 .
$$

for a flat background the scale factor $\ell$, energy density $\mu$ and expansion $\Theta$ are given by:

$$
\ell \propto \eta^{\frac{2}{3 \gamma-2}}, \quad \mu \propto \eta^{-\frac{6 \gamma}{3 \gamma-2}}, \quad \Theta \propto \eta^{-\frac{3 \gamma}{3 \gamma-2}} .
$$

Substituting the background parameters into (25) and (26), and integrating, gives the following general solutions

$$
\sigma_{(k)}=\eta^{\frac{3 \gamma-10}{2(3 \gamma-2)}}\left[J_{\nu}(k \eta) C_{(k)}^{(1)}+N_{\nu}(k \eta) C_{(k)}^{(2)}\right],
$$

and

$$
H_{(k)}=\eta^{\frac{3 \gamma-14}{2(3 \gamma-2)}}\left[\left[J_{\nu}(k \eta) D_{(k)}^{(1)}+N_{\nu}(k \eta) D_{(k)}^{(2)}\right],\right.
$$

where $C$ and $D$ are arbitrary constants and $J_{\nu}, N_{\nu}$ denote the Bessel functions of the first and second kind.

For large scales $(k \rightarrow 0)$, we obtain power - law solutions:

$$
\sigma_{(k)}=\eta^{\frac{3 \gamma-4}{3 \gamma-2}} C_{(k)}^{(1)}+\eta^{-\frac{6}{3 \gamma-2}} C_{(k)}^{(2)} .
$$

and

$$
H_{(k)}=D_{(k)}^{(1)} \eta^{\frac{3(\gamma-2)}{3 \gamma-2}}+D_{(k)}^{(2)} \eta^{-\frac{8}{3 \gamma-2}} .
$$

In the case of a flat background, the third order equation for $E_{(k)}$ (17) becomes:

$$
\begin{aligned}
E_{(k)}^{\prime \prime \prime} & +(4+3 \gamma) \frac{\ell^{\prime}}{2 \ell} E_{(k)}^{\prime \prime}+\left[(4 \gamma+6) \mu \ell^{2}+k^{2}\right] E_{(k)}^{\prime} \\
& +\left\{\frac{1}{6}[16-\gamma(3 \gamma-2)] \mu \ell^{2}+\frac{3}{2} \gamma k^{2}\right\} \ell \Theta E_{(k)}=0
\end{aligned}
$$


This time, a general solution could not be found, however for large scale modes we obtain:

$$
E_{(k)}=\eta^{-\frac{3(\gamma+2)}{3 \gamma-2}} E_{(k)}^{(1)}+\eta^{-\frac{4}{3 \gamma-2}} E_{(k)}^{(2)}+\eta^{\frac{3 \gamma-8}{3 \gamma-2}} E_{(k)}^{(3)} .
$$

The first two of these modes were previously known [9, 10]. The third one, due to the third order nature of the equation for $E_{(k)}$, has to be unphysical as it is not a solution of the original system of first order equations. The point is an essential one: one cannot satisfy one or other of these equations in isolation, one has to solve the set as a whole. In this case, we can solve first for $\sigma_{a b}$ (a second order equation), as above, and then determine $E_{a b}$ from (16) by simply differentiating this solution. This will determine solutions to the third order equation for $E_{a b}$; but there will be two modes, not three:

$$
E_{(k)}=\eta^{-\frac{3(\gamma+2)}{3 \gamma-2}} E_{(k)}^{(1)}+\eta^{-\frac{4}{3 \gamma-2}} E_{(k)}^{(2)} .
$$

This shows that in fact the effective equation governing the solution for $E_{a b}$, considered as a solution to the set of equations as a whole, is second order, corresponding to the previously known solutions, even though one apparently cannot write down a separate second order equation for $E_{a b}$ alone.

\section{The cosmological context}

We have emphasized here that we are considering the problem of gravitational waves in a cosmological setting.

\subsection{Averaging}

The key point in the analysis has been a non-vacuum assumption. Is this realistic?

As discussed above, the evolution equations for $E_{a b}$ and $H_{a b}$ are the same when $\mu+p=0$. Now for astrophysical sources of gravitational waves, such as pulsars or merging binary systems in our own galaxy, the vacuum assumption is likely to be good for much of the region the wave travels in, since the intergalactic and interstellar media are relatively tenuous. Waves with wavelength less than $1 \mathrm{Mpc}$ will in fact travel through a vacuum for much of the time; and those of wavelengths less than $1 \mathrm{pc}$ will be in an effective vacuum for almost all the time. Thus in this case the Hawking analysis will be adequate; both $E_{a b}$ and $H_{a b}$ will satisfy the same second-order wave equation.

However cosmological scale gravitational waves, and certainly those with wavelengths of the order of the horizon size, will experience a non-vacuum spacetime background all the time. Thus our problem is one of averaging: on what scale does the geometry of the universe start to approximate that of a 
Robertson- Walker universe? [7] On smaller scales, the vacuum approximation will be acceptable for much but not all the time; on larger scales it is not, and the third order equation discussed above for $E_{a b}$ applies.

In particular, we can ask the following question: What happens to $E_{a b}$ for small-scale waves if, after traveling in vacuum, it comes across a non-zero matter concentration, for example in the case of a gravitational wave crossing a galaxy cluster? The wave equation for the waves will change then from second to third order. However perhaps this is not too drastic, as the effective order of the set of equations will remain second order, as discussed above. Nevertheless the junction conditions at such a change need careful consideration, because the standard assumptions allow a discontinuity of the curvature tensor at a boundary of this kind. We will not pursue this further here, but note it as worthy of investigation.

\subsection{Sharp phase transitions}

The same kind of issue arises in the early universe, in a different context.

If the early universe went through an inflationary phase, then there must have been a transition at the end of inflation from the de-Sitter phase to a radiation dominated phase, often treated as an instantaneous 3 -surface, although in reality reheating is not instantaneous. The question we pose is as follows: can there be amplification of perturbations across this boundary? The standard answer is that density perturbations can be amplified, but that tensor perturbations cannot. This is simply a result of the Darmois junction conditions which require that the three-metric and the extrinsic curvature of the three-metric be continuous across the boundary.

The key point for us is that for tensor perturbations, this theorem of noamplification does not carry through in the covariant case since both the electric and magnetic parts of the Weyl tensor will depend on the second derivatives of the metric perturbations, which are unconstrained by the junction conditions. Thus we cannot agree with the conclusion that gravitational waves cannot be amplified across a sharp phase transition. We will not discuss this issue further here; it is raised because it is similar to the situation just discussed.

\section{Conclusion}

It is interesting that the evolution equations for $E_{a b}$ and $H_{a b}$ are so different, because this implies a radical break with the analogy of source-free electromagnetism, which had been thought to carry through almost completely, at least qualitatively. This is assuming of course that the evolution equation for $E$ cannot be reduced to second order (the 'hidden symmetry' option). We have not

found a way to do so; the third order equation appears to us to be genuinely third order. 
Clearly it is interesting to locate the conditions when the evolution equations for $E_{a b}$ and $H_{a b}$ reduce to the same second order equation. We found that this happens (i) when $(\mu+p)=0$, i.e. in vacuum and in de Sitter spacetime, (ii) for the exceptional equation of state $p=-(1 / 3) \mu$, (iii) and the unphysical variation of $\gamma$ given by (24). We exclude all these cases in a realistic cosmological context, for long-wavelength waves.

Our analysis shows that for large scale gravitational waves in a universe model with realistic matter, $E_{a b}$ and $H_{a b}$ obey quite different equations, yet these are consistent with each other. This is like the situation for Maxwell's equations with a source, as is shown in the accompanying paper [8]. Although the wave equation for $E_{a b}$ is third order, it has the same characteristics as the second order equation for $H_{a b}$, and both can be solved from a single 'potential', namely the shear (obeying a second order wave equation). This presumably is

how the Bardeen analysis leads only to a second order equation: that equation is also for a potential, and the equation satisfied by the Weyl tensor itself is not written down.

\section{References}

[1] Bardeen J M 1980 Phys Rev D 22, 1882.

[2] Bruni M, Dunsby P K S and Ellis G F R 1992 Ap. J., 395, 34.

[3] Dunsby P K S and Ellis G F R 1996 "Covariant Characterisation of Almost Friedmann- Robertson-Walker Cosmologies", University of Cape Town preprint.

[4] J. Ehlers, Abh. Mainz Akad. Wiss. Lit. (Math. Nat. Kl.) 11, 1 (1961).

[5] Ellis G F R "Relativistic Cosmology" in Cargèse Lectures in Physics, vol VI, ed. E. Schatzmann (Gordon and Breach, 1973).

[6] Ellis G F R and Bruni M 1989 Phys. Rev. D 40, 1804.

[7] Ellis G F R and Stoeger W R 1987 Class. Quantum Gravit. 4, 1679.

[8] Ellis G F R and Hogan P A 1996 "The Electromagnetic Analogue of some Gravitational Perturbations in Cosmology", University of Cape Town preprint.

[9] Goode S W 1989 Phys. Rev. D, 39, 2992.

[10] Goode S W 1983 "Spatially inhomogeneous cosmologies and their relation with the Friedmann-Robertson - Walker cosmologies" Ph.D. thesis, University of Waterloo.

[11] Hawking S W 1966 Ap. J. 145, 544. 
[12] Harrison E R 1967 Rev. Mod. Phys. 39, 862.

[13] Hogan P A and Ellis G F R 1995 "Propogation of Information by Electromagnetic and Gravitational Waves in Cosmology", University of Cape Town preprint.

[14] Lifshitz, E M 1946 Phys USSR 10, 116.

[15] Lesame W M, Dunsby P K S and Ellis G F R 1995 Phys. Rev. D 52, 3406.

[16] Lesame W M, Ellis G F R and Dunsby P K S 1996 Phys. Rev. D 53, 738. 